CU-TP-601

June 1993

\title{
Hard Thermal Loops, Gauged WZNW-Action and the Energy of Hot Quark-Gluon Plasma
}

\author{
V.P.Nair * \\ Physics Department, Columbia University \\ New York, NY 10027
}

\begin{abstract}
The generating functional for hard thermal loops in Quantum Chromodynamics is rewritten in terms of a gauged Wess-Zumino-Novikov-Witten action by introducing an auxiliary field. This shows in a simple way that the contribution of hard thermal loops to the energy of the quark-gluon plasma is positive.
\end{abstract}

This research was supported in part by the U.S. Department of Energy.

* Address after September 1, 1993: Physics Department, City College of the CUNY, Convent Avenue at 138 Street, New York, NY 10031. 
The effective action or generating functional $\Gamma$ for hard thermal loops in Quantum Chromodynamics (QCD) has been the subject of many recent investigations [1-4]. We have shown that $\Gamma$ is essentially given by the eikonal for a Chern-Simons (CS) gauge theory [3]. $\Gamma$ is a nonlocal functional of the gauge potential $A_{\mu}$ and leads to new effective propagators and vertices. These effective propagators and vertices help to carry out the reorganization (and partial summation) of thermal perturbation theory necessary to ensure that all contributions to a given order in the coupling constant are consistently included. Further, many of the properties of the quark-gluon plasma at high temperature can be understood in terms of the action

$$
\mathcal{S}=\int \frac{1}{4} F^{2}+\Gamma[A]
$$

where we add $\Gamma$ to the usual Yang-Mills action. The action (1) gives a gauge invariant description of Debye screening and plasma waves. Also, with a proper $i \epsilon$-prescription, the equations of motion following from (1) (which are also nonlocal) can describe Landau damping [4]. Clearly further investigation of $\Gamma$ is interesting both for the programme of resummation of perturbation theory and for clarifying properties of the quark-gluon plasma at high temperature.

In this paper, we introduce an auxiliary field and rewrite $\Gamma$ in terms of a gauged Wess-Zumino-Novikov-Witten (WZNW) theory. This field is actually defined on a sixdimensional space. The elimination of the auxiliary field via its equations of motion leads back to (1) with $\Gamma$ expressed in terms of the potentials. The introduction of the auxiliary field has the advantage that the equations of motion are local. This facilitates further analysis since the quark-gluon plasma (at high temperature) can now be described by a gauge theory coupled, in a local and more or less standard way, to an auxiliary field. We also obtain the energy functional and show that it is positive for all physical field configurations, a question which has been of some concern recently [5]. This rewriting of the theory may also help in setting up the resummed perturbation theory; new auxiliary field propagators and vertices can be used to incorporate hard thermal loop effects.

We begin by recalling some of the essential results of ref.[3]. $Q, Q^{\prime}$ will denote the null vectors $Q_{\mu}=(1, \vec{Q}), \quad Q_{\mu}^{\prime}=(1,-\vec{Q})$ with $\vec{Q} \cdot \vec{Q}=1$. We introduce the light- 
cone coordinates $u=\frac{1}{2}\left(Q^{\prime} \cdot x\right), v=\frac{1}{2}(Q \cdot x)$; the Euclidean version of $Q^{\prime} \cdot x$ and $Q \cdot x$ will be denoted by $z, \bar{z}$ respectively. The corresponding components of the potential are $A_{+}=\frac{1}{2} A \cdot Q, A_{-}=\frac{1}{2} A \cdot Q^{\prime}$, with $A_{+} \rightarrow A_{z}, A_{-} \rightarrow A_{\bar{z}}$ in the Euclidean case. Here $A_{\mu}=-i t^{a} A_{\mu}^{a} ; t^{a}$ are hermitian matrices representing the generators of the Lie algebra of the gauge group, which we take to be $S U(N)$.

We shall, for simplicity of presentation, introduce the auxiliary field in its Euclidean version first. The effective action $\Gamma$ can be written as

$$
\Gamma=-\frac{k}{\pi} \int d \Omega d^{2} \mathbf{x}^{T}\left[\int d^{2} z \operatorname{tr}\left(A_{z} A_{\bar{z}}\right)+i \pi I\left(A_{z}\right)+i \pi I\left(A_{\bar{z}}\right)\right]
$$

where $k=\left(N+\frac{1}{2} N_{F}\right) \frac{T^{2}}{6} ; N_{F}$ is the number of quark flavors and $T$ is the temperature. The integration $\int d \Omega$ in (2) is over the angular directions of $\vec{Q} \cdot \mathbf{x}^{T}$ denotes the two coordinates transverse to $\vec{Q}$, i.e. $\vec{Q} \cdot \mathbf{x}^{T}=0$. $I\left(A_{z}\right)$ in (2) is the eikonal for a CS theory defined by

$$
I=i \sum_{2}^{\infty} \frac{(-1)^{n}}{n} \int \frac{d^{2} z_{1}}{\pi} \cdots \frac{d^{2} z_{n}}{\pi} \frac{\operatorname{tr}\left(A_{z}\left(x_{1}\right) \cdots A_{z}\left(x_{n}\right)\right)}{\bar{z}_{12} \cdots \bar{z}_{n-1 n} \bar{z}_{n 1}}
$$

$\left(\bar{z}_{i j}=\bar{z}_{i}-\bar{z}_{j}.\right)$ All $A$ 's have the same argument for the transverse coordinates $\mathbf{x}^{T} . I\left(A_{z}\right)$ has the property that

$$
\delta I\left(A_{z}\right)=\frac{i}{\pi} \int d^{2} z \operatorname{tr}\left(a_{\bar{z}} \delta A_{z}\right)
$$

where $a_{\bar{z}}$ obeys the equation

$$
\partial_{z} a_{\bar{z}}-\partial_{\bar{z}} A_{z}+\left[A_{z}, a_{\bar{z}}\right]=0
$$

$I\left(A_{\bar{z}}\right)$ in $(2)$ is obtained from $I\left(A_{z}\right)$ by $Q \leftrightarrow Q^{\prime}$. Equation (4b) has the following solution for $a_{\bar{z}}$.

$$
a_{\bar{z}}=\sum_{1}^{\infty}(-1)^{n-1} \int \frac{d^{2} z_{1}}{\pi} \cdots \frac{d^{2} z_{n}}{\pi} \frac{A_{z}\left(x_{1}\right) \cdots A_{z}\left(x_{n}\right)}{\left(\bar{z}-\bar{z}_{1}\right) \bar{z}_{12} \cdots\left(\bar{z}_{n}-\bar{z}\right)}
$$

$I\left(A_{z}\right)$ can also be related to the chiral Dirac determinant in two dimensions and to the WZNW-action [6]. It is possible to introduce an $S L(N, \mathbf{C})$-matrix $U$ such that $A_{z}=-\partial_{z} U U^{-1}$ and $A_{\bar{z}} \equiv-\left(A_{z}\right)^{\dagger}=U^{\dagger}{ }^{-1} \partial_{\bar{z}} U^{\dagger}$. The eikonal $I\left(A_{z}\right)$ can then be written as $I=i \log \operatorname{det}\left(\partial_{z}+A_{z}\right)=-i S(U) . S(U)$ is the WZNW-action [7]

$$
S(U)=\frac{1}{2 \pi} \int d^{2} z \operatorname{tr}\left(\partial_{z} U \partial_{\bar{z}} U^{-1}\right)-\frac{i}{12 \pi} \int d^{3} x \epsilon^{\mu \nu \alpha} \operatorname{tr}\left(U^{-1} \partial_{\mu} U U^{-1} \partial_{\nu} U U^{-1} \partial_{\alpha} U\right)
$$


$S$ obeys the Polyakov-Wiegmann (PW) composition rule [6]

$$
S(h g)=S(h)+S(g)-\frac{1}{\pi} \int d^{2} z \operatorname{tr}\left(h^{-1} \partial_{\bar{z}} h \partial_{z} g g^{-1}\right)
$$

In terms of $U$, we may in fact write $\Gamma$ as

$$
\Gamma=-k \int d \Omega d^{2} \mathbf{x}^{T} S\left(U^{\dagger} U\right)
$$

Using $\Gamma$ as given by (2) in the action (1), we can write down the equations of motion for the gauge field. (Equation (4a) or the PW-property (7) can be used for the variation of the action.) We find the equations of motion

$$
\left(D_{\mu} F^{\mu \nu}\right)^{a}+\frac{k}{2 \pi} \int d \Omega \operatorname{tr}\left\{\left(-i t^{a}\right)\left[\left(a_{\bar{z}}-A_{\bar{z}}\right) Q^{\nu}+\left(a_{z}-A_{z}\right) Q^{\prime \nu}\right]\right\}=0
$$

(This equation was given in ref.[4].) Notice that we may regard $a_{\bar{z}}, a_{z}$ as new fields which are determined by equation (4b) and its conjugate. The solution (5) and its conjugate give the current in (9).

We now introduce an auxiliary field $M(x, \vec{Q})$ which is a complex $N \times N$ matrix of unit determinant. It is defined on $\mathcal{M} \times S^{2}$ where $\mathcal{M}$ is spacetime and $S^{2}$ is the two-sphere corresponding to the orientations of $\vec{Q}$. In other words $M(x, \vec{Q}): \mathcal{M} \times S^{2} \rightarrow S L(N, \mathbf{C})$. We also define $H=M^{\dagger} M$.

The action for the fields $A_{\mu}^{a}(x), M(x, \vec{Q})$ is taken to be

$$
\mathcal{S}=\int \frac{1}{4} F^{2}+k \int d \Omega d^{2} \mathbf{x}^{T} \tilde{S}(M, A)
$$

$\tilde{S}(M, A)$ is the gauged WZNW-action given by $[8]$

$$
\tilde{S}(M, A)=S(H)+\frac{1}{\pi} \int d^{2} z \operatorname{tr}\left(H^{-1} \partial_{\bar{z}} H A_{z}-A_{\bar{z}} \partial_{z} H H^{-1}+A_{z} H^{-1} A_{\bar{z}} H-A_{z} A_{\bar{z}}\right)
$$

$\tilde{S}(M, A)$ is invariant under the gauge transformation

$$
\begin{gathered}
M \rightarrow M^{\prime}=M h^{-1}, \quad H \rightarrow H^{\prime}=h H h^{-1} \\
A_{\mu} \rightarrow A_{\mu}^{\prime}=h A_{\mu} h^{-1}-\partial_{\mu} h h^{-1}
\end{gathered}
$$


where $h \in S U(N)$ is a function of $x(\operatorname{not} \vec{Q})$. $\tilde{S}(M, A)$ can be written in terms of $U$ defined by $A_{z}=-\partial_{z} U U^{-1}$, by using the PW-property (7), as

$$
\tilde{S}(M, A)=S\left(U^{\dagger} H U\right)-S\left(U^{\dagger} U\right)
$$

This makes the gauge invariance property manifest.

We shall now show that the elimination of the field $M(x, \vec{Q})$ leads back to the equation of motion (9) and the expression (8) for $\Gamma$. The equations of motion for the effective action $(10,11)$ can be easily obtained using $(7)$. We find

$$
\begin{gathered}
\partial_{z} \mathcal{A}_{\bar{z}}-\partial_{\bar{z}} A_{z}+\left[A_{z}, \mathcal{A}_{\bar{z}}\right]=0 \\
\partial_{\bar{z}} \mathcal{A}_{z}-\partial_{z} A_{\bar{z}}+\left[A_{\bar{z}}, \mathcal{A}_{z}\right]=0 \\
\left(D_{\mu} F^{\mu \nu}\right)^{a}+\frac{k}{2 \pi} \int d \Omega \operatorname{tr}\left\{\left(-i t^{a}\right)\left[\left(\mathcal{A}_{\bar{z}}-A_{\bar{z}}\right) Q^{\nu}+\left(\mathcal{A}_{z}-A_{z}\right) Q^{\prime \nu}\right]\right\}=0
\end{gathered}
$$

where

$$
\begin{aligned}
& \mathcal{A}_{z}=H A_{z} H^{-1}-\partial_{z} H H^{-1} \\
& \mathcal{A}_{\bar{z}}=H^{-1} A_{\bar{z}} H+H^{-1} \partial_{\bar{z}} H
\end{aligned}
$$

Equations $(14 \mathrm{a}, 14 \mathrm{~b})$ are in fact the same. They can be solved by $\mathcal{A}_{\bar{z}} \equiv H^{-1} A_{\bar{z}} H+$ $H^{-1} \partial_{\bar{z}} H=a_{\bar{z}}$, the power series solution of (5). (This must be regarded as a solution for $M(x, \vec{Q})$ or $H$. The explicit form for $H$ will be fairly complicated; we do not need it.) Using this solution, we see that (15) does indeed reduce to (9), showing that the action (10) is an acceptable rewriting of the theory of equations $(1,2)$. In terms of the matrix $U$, the solution for $H$ is $U^{-1} U^{\dagger-1}$ or $M(x, \vec{Q})=U^{\dagger-1}$. Using this in (13), we see that

$$
\mathcal{S}=\int \frac{1}{4} F^{2}-k \int d \Omega d^{2} \mathbf{x}^{T} S\left(U^{\dagger} U\right)
$$

recovering $\Gamma$ in the form (8).

The equation of motion for the gauge field, viz. equation (15), can also be written as

$$
\left(D_{\mu} F^{\mu \nu}\right)^{a}+\frac{k}{2 \pi} \int d \Omega \operatorname{tr}\left\{\left(-i t^{a}\right)\left[H^{-1} D_{\bar{z}} H Q^{\nu}-D_{z} H H^{-1} Q^{\prime \nu}\right]\right\}=0
$$

where $D_{\mu}$ is the gauge covariant derivative, $D_{\mu} H=\partial_{\mu}+\left[A_{\mu}, H\right]$. 
The action (11) involves the Wess-Zumino (WZ) term, the second term on the right hand side of (6) with $U \rightarrow H$, which requires an extension of $H$ into a higher dimensional space $\tilde{\mathcal{M}}$. However, as discussed in ref.[3], the topology of hermitian matrices being trivial, there is no argument for the quantization of the coefficient $k$. In particular, we can find a parametrization for $H$, e.g. $H=e^{\theta}, \theta$ being hermitian and traceless, such that the WZ-term can be written as an integral over spacetime rather than $\tilde{\mathcal{M}}$. Put another way, $\theta$ can be thought of as $\log H$. In general, there are ambiguities related to the choice of the branch of the logarithm which can eventually lead to the quantization requirement on $k$. In the present case, the hermiticity of $\theta$ eliminates the branch ambiguities.

We now consider the auxiliary field and action in Minkowski space. In the Euclidean case, the solution for $H$ is of the form $U^{-1} U^{\dagger-1}$ where $A_{z}=-\partial_{z} U U^{-1}, A_{\bar{z}}=$ $-\partial_{\bar{z}}\left(U^{\dagger-1}\right) U^{\dagger}$. With Minkowski signature, we can write

$$
A_{+}=-\partial_{+} V V^{-1}, \quad A_{-}=-\partial_{-} V^{\prime} V^{\prime-1}
$$

Evidently, $V^{\prime}(x, \vec{Q})=V(x,-\vec{Q}) . \quad V(x, \vec{Q})$ can be taken to be unitary. The comparison with the Euclidean version suggests the introduction of an auxiliary field $N(x, \vec{Q})$ with $G=N^{\dagger}(x,-\vec{Q}) N(x, \vec{Q})$ playing the role of $H$. The correspondence is thus

$$
M \leftrightarrow N(x, \vec{Q}), \quad M^{\dagger} \leftrightarrow N^{\dagger}(x,-\vec{Q}), \quad H \leftrightarrow G
$$

$M(x, \vec{Q})$ is not unitary but $N(x, \vec{Q})$ is an $S U(N)$-matrix. $G$ is also special unitary and from its definition is seen to satisfy the condition $G^{\dagger}(x, \vec{Q})=G(x,-\vec{Q})$.

The action in Minowski space is taken as

$$
\begin{gathered}
\mathcal{S}=\int-\frac{1}{4} F^{2}+k \int d \Omega\left[\int d^{2} \mathbf{x}^{T} S(G)+\frac{1}{\pi} \int d^{4} x \operatorname{tr}\left(G^{-1} \partial_{-} G A_{+}-A_{-} \partial_{+} G G^{-1}\right.\right. \\
\left.\left.+A_{+} G^{-1} A_{-} G-A_{+} A_{-}\right)\right]
\end{gathered}
$$

where $S(G)$ is given by (6) with $U \rightarrow G$ and $\partial_{+}, \partial_{-}$replacing $\partial_{z}, \partial_{\bar{z}}$. The equations of motion are now given by

$$
\partial_{+} \mathcal{A}_{-}-\partial_{-} A_{+}+\left[A_{+}, \mathcal{A}_{-}\right]=0
$$




$$
\begin{gathered}
\left(D_{\mu} F^{\mu \nu}\right)^{a}-J^{\nu a}=0 \\
J^{\nu a}=-\frac{k}{2 \pi} \int d \Omega \operatorname{tr}\left\{\left(-i t^{a}\right)\left[\left(\mathcal{A}_{-}-A_{-}\right) Q^{\nu}+\left(\mathcal{A}_{+}-A_{+}\right) Q^{\prime \nu}\right]\right\} \\
=-\frac{k}{2 \pi} \int d \Omega \operatorname{tr}\left\{\left(-i t^{a}\right)\left[G^{-1} D_{-} G Q^{\nu}-D_{+} G G^{-1} Q^{\prime \nu}\right]\right\}
\end{gathered}
$$

Again, solving equation (22a) and substituting in (22b), we can recover the Minkowski version of equation (9). In solving equation (22a), we need to specify certain analyticity properties or an $i \epsilon$-prescription to define the inverse of $\partial_{+}$. As we have discussed in ref. [4], this is a retardation property. (The expression for the current in (23) is also similar to what was given in the ref.[4]; the difference is that here $G$ is an independent field to begin with.)

As in the Euclidean case, the WZ-term seems to require an extension of $G$ into a higher dimensional space. $G$ is now unitary and one might worry that this would lead to a quantization requirement on $k$ so that physical results remain the same for different extensions of $G$ into the higher dimensional space. Actually we do not expect such a condition. $G$ is not any unitary matrix; it has the property $G^{\dagger}(x, \vec{Q})=G(x,-\vec{Q})$. Thus $\log G$ must be odd under $\vec{Q} \rightarrow-\vec{Q}$. This condition will eliminate some of the branch ambiguities so that the WZ-term can again be written as an integral over spacetime. Alternatively, existence of the Euclidean continuation via rules (20) show that $k$ is not quantized. Notice also that the fields $A_{\mu}^{a}(x), N(x, \vec{Q})$ obey local equations of motion.

We now turn to the energy functional. One could start with the action (21), introduce phase space variables, Poisson brackets, etc. However, there is a simpler way to obtain the energy functional. We consider the action for a finite interval of time, say from zero to $t$, and use the fact that the Hamiltonian or energy can be defined as $\mathcal{H}=-\frac{\partial \mathcal{S}}{\partial t}$. As an example consider the theory of a scalar field $\phi$ with an action

$$
\mathcal{S}=\int_{0}^{t} d t d^{3} x\left[\frac{1}{2}\left(\frac{\partial \phi}{\partial t}\right)^{2}-\frac{1}{2}(\nabla \phi)^{2}-V(\phi)\right]
$$

If we divide the interval $[0, t]$ into $n$ segments, with $t_{0}=0, t_{n}=t$ and write

$$
\mathcal{S}=\int d^{3} x\left[\frac{1}{2} \sum \frac{\left(\phi_{n-i}-\phi_{n-i-1}\right)^{2}}{\left(t_{n-i}-t_{n-i-1}\right)}-\left(\frac{1}{2}(\nabla \phi)^{2}+V(\phi)\right)_{n-i}\left(t_{n-i}-t_{n-i-1}\right)\right]
$$


we see immediately that

$$
\mathcal{H} \equiv-\frac{\partial \mathcal{S}}{\partial t_{n}}=\int d^{3} x\left[\frac{1}{2}\left(\frac{\partial \phi}{\partial t}\right)^{2}+\frac{1}{2}(\nabla \phi)^{2}+V(\phi)\right]
$$

as $t_{n-i}-t_{n-i-1} \rightarrow 0, n \rightarrow \infty$. This is of course as expected. For the present case, the WZ-term in (21) has only one time-derivative; it does not depend on $\left(t_{n}-t_{n-1}\right)$ and so does not contribute to the energy. From the rest of the terms in $\mathcal{S}$, we see immediately that

$$
\mathcal{H}=\int d^{3} x\left\{\frac{E^{2}+B^{2}}{2}+\frac{k}{8 \pi} \int d \Omega \operatorname{tr}\left[\left(D_{0} G D_{0} G^{-1}\right)+\left(\vec{Q} \cdot \vec{D} G \vec{Q} \cdot \vec{D} G^{-1}\right)\right]-A_{0}^{a} \mathcal{G}^{a}\right\}
$$

where $F_{0 i}^{a}=E_{i}^{a}, F_{i j}^{a}=\epsilon_{i j k} B_{k}^{a}$ and

$$
\mathcal{G}^{a}=(\vec{D} \cdot \vec{E})^{a}+\frac{k}{8 \pi} \int d \Omega \operatorname{tr}\left[\left(-i t^{a}\right)\left(G^{-1} D_{-} G-D_{+} G G^{-1}\right)\right]
$$

$\mathcal{G}^{a}=0$ is the Gauss law of the theory. It is the time-component of the equation of motion (22b). From (27), we see that $\mathcal{H}$ is positive for all physical field configurations, viz. for those which satisfy the Gauss law.

In the action and the equations of motion, it is only the field combination $G$ which appears. One could take this to be the basic field instead of $N(x, \vec{Q})$, but the condition

$G^{\dagger}(x, \vec{Q})=G(x,-\vec{Q})$ has to be imposed so as to ensure that there are no ambiguities in the extensions of $G$ required for the WZ-term.

\section{References}

1. E.Braaten and R.Pisarski, Phys.Rev.D 42, 2156 (1990); Nucl.Phys. B337, 569 (1990); ibid. B339, 310 (1990); Phys.Rev. D 45, 1827 (1992).

2. J.Frenkel and J.C.Taylor, Nucl.Phys. B334, 199 (1990); J.C.Taylor and S.M.H.Wong, Nucl.Phys. B346, 115 (1990).

3. R.Efraty and V.P.Nair, Phys.Rev.Lett. 68, 2891 (1992); Columbia Preprint CU-TP 579, November 1992 (to be published in Phys.Rev.D). 
4. R.Jackiw and V.P.Nair, Columbia-MIT Preprint CU-TP 594, CTP \# 2205, April 1993 (submitted to Phys.Rev.D).

5. F.T.Brandt, J.Frenkel and J.C.Taylor, Cambridge University Preprint DAMTP-93-02, February 1993.

6. A.M.Polyakov and P.B.Wiegmann, Phys.Lett. 141B, 223 (1984); D.Gonzales and A.N.Redlich, Ann.Phys. 169, 104 (1986); G.V.Dunne, R.Jackiw and C.A.Trugenberger, Ann.Phys. 194, 197 (1989).

7. S.P.Novikov, Usp.Mat.Nauk 37, 3 (1982); E.Witten, Commun.Math.Phys. 92, 455 (1984).

8. R.I.Nepomechie, Phys.Rev.D 33, 3670 (1986); D.Karabali, Q-H.Park, H.J.Schnitzer and Z.Yang, Phys.Lett. 216B, 307 (1989); D.Karabali and H.J.Schnitzer, Nucl.Phys. B329, 649 (1990); K.Gawedzki and A.Kupianen, Phys.Lett. 215B, 119 (1988); Nucl.Phys. B320, 649 (1989). 